\title{
Температурно-зависимое интегральное поглощение экситонных поляритонов в полупроводниковых кристаллах
}

\author{
(C) Р.П. Сейсян, С.А. Ваганов \\ Физико-технический институт им. А.Ф. Иофрфе Российской академии наук, \\ 194021 Санкт-Петербург, Россия \\ " E-mail: sv.exciton@mail.ioffe.ru
}

Поступила в Редакцию 22 октября 2019 г.

В окончательной редакции 28 ноября 2019 г.

Принята к публикации 2 декабря 2019 г.

Данная работа выделяет экспериментальное использование температурного фактора при изучении интегрального поглощения как метода экспериментального подтверждения и исследования механизма экситон-поляритонного светопереноса вблизи края фундаментального поглощения в полупроводниковых кристаллах с пространственной дисперсией. Обобщены результаты экспериментальных исследований температурно-зависимого интегрального экситонного поглощения, приведены экспериментально определенные значения критической температуры, выше которой интегральное поглощение становится постоянным, соответствующие ему значения критического параметра затухания и продольно-поперечного расщепления для исследованных авторами полупроводников CdTe, GaAs, InP, ZnSe, ZnTe.

Ключевые слова: экситонное поглощение, интегральное поглощение, температурно-зависимое поглощение, экситонный поляритон, полупроводниковые кристаллы.

DOI: 10.21883/FTP.2020.04.49135.9293

\section{1. Введение}

Интегральное поглощение в спектроскопии широко используется для определения силы осциллятора в диссипативных системах, в которых поглощаемая в максимуме энергия обратно пропорциональна параметру затухания (осциллятор Лоренца). Непостоянство интеграла коэффициента поглощения $\alpha(\omega) \quad K=\int_{0}^{\infty} \alpha(\omega) d \omega$ указывает на изменение закона поглощения энергии, связанное либо с изменением дисперсионных характеристик осцилляторов, либо с появлением между ними взаимодействия. В случае экситон-поляритонного светопереноса в полупроводниковых кристаллах начальный рост интегрального поглощения экситона $K=K(\Gamma)$ при увеличении параметра затухания $\Gamma[1]$,

$$
K(\Gamma)=\frac{2 K_{\max }}{\pi}\left[\arctan \frac{\frac{\Gamma}{\Gamma_{c}}}{\sqrt{1-\left(\frac{\Gamma}{\Gamma_{c}}\right)^{2}}}+\frac{\Gamma}{\Gamma_{c}} \sqrt{1-\left(\frac{\Gamma}{\Gamma_{c}}\right)^{2}}\right],
$$

объясняется двухмодовым характером распространения волн из-за эффекта пространственной дисперсии, обусловленного конечной трансляционной массой экситона [2]. После достижения критического значения параметра затухания $\Gamma>\Gamma_{c}$ светоперенос фактически осуществляется только одной волной, описываемой дисперсионным соотношением, не зависящим от волнового вектора, и интегральное поглощение становится постоянным $K=K_{\max }$.

В полупроводниковых кристаллах экситонные поляритоны распространяются без истинного поглощения света, поглощение энергии происходит только при нерадиа- ционном распаде экситонов в результате взаимодействия с диссипативной подсистемой [3,4]. Среди множества факторов, изменяющих параметр затухания экситонных поляритонов, температурный является наиболее экспериментально доступным и воспроизводимым.

Зависимость экситонного поглощения от температуры экспериментально изучалась в большом количестве различных полупроводниковых кристаллов $\mathrm{CdS}[5,6]$, GaAs [7,8], CdTe, ZnTe [9], ZnSe [10,11], GaSe [12], $\mathrm{GaN}$ [13], ZnS, ZnTe [11], $\mathrm{ZnP}_{2}$ [14], $\mathrm{PbI}_{2}$ [15], InP [16]. По сравнению с фотолюминесценцией, экспериментальный метод исследования экситон-поляритонного переноса регистрацией светопропускания через кристалл менее чувствителен к качеству образцов, особенно к наличию дефектов, примесей, и состоянию приповерхностного слоя, но требует использования сверхтонких образцов, свободных от неоднородных деформаций и остаточных напряжений, которые приводят к значительному неоднородному уширению экситонных линий.

Аномальный рост интегрального экситонного поглощения при низких температурах отмечен в работе [6] при исследовании монокристалов $\mathrm{CdS}$ при помощи высокочувствительной регистрирующей аппаратуры высокого спектрального разрешения.

Следующее за ростом насыщение интегрального экситонного поглощения при температуре выше критической $T>T_{c}$ было впервые экспериментально продемонстрировано в высококачественных образцах GaAs [7] при помощи контурного анализа, позволившего изолировать экситонные линии из суммарного спектра края фундаментального поглощения при высоких температуpax. В дальнейшем температурно-зависимое интегральное поглощение было исследовано в CdTe, ZnTe [9], 
$\mathrm{ZnSe}$ [10], $\mathrm{ZnP}_{2}$ [14], InP [16], а также в твердых растворах [17] и гетероструктурах с множественными квантовыми ямами [18].

Цель работы заключалась в обобщении результатов экспериментальных исследований зависимости интегрального поглощения экситона в полупроводниковых кристаллах, интерпретируемой с помощью рассмотренного в работе [1], экситон-поляритонного механизма светопереноса в средах с пространственной дисперсией, при использовании температуры в качестве фактора, изменяющего параметр затухания экситонных поляритонов.

\section{2. Образцы и техника экспериментального исследования}

В полупроводниковых кристаллах с сильным экситонным поглощением экспериментальное изучение спектров пропускания возможно только в тонких образцах высокого кристаллофизического совершенства, так как воздействие эффектов примесной ионизации, штарковского сдвига экситона в поле заряда поверхностных состояний, локализация, размерное квантование экситона как целого и составляющих его носителей в приповерхностных ямах может существенно модифицировать структуру экситонного спектра.

Свободные от неоднородных деформаций и остаточных напряжений открытые слои полупроводниковых кристаллов получались механическим и химическим утончением.

Также использовались образцы полупроводниковых кристаллов с интерфейсной границей, выращенные методом молекулярно-пучковой эпитаксии. На высокосовершенной границе раздела слоя исследуемого полупроводника внутри более широкозонного материала практически отсутствует заряд, но могут возникать напряжения, связанные с различием постоянных решетки материалов интерфейса, и явления, обусловленные флуктуациями ширины слоя и сплавным беспорядком в материалах, окружающих слой.

Экспериментальные образцы переносились в бокс из покровного стекла и размещались свободно без применения клеевой и механической фиксации для исключения возникновения неконтролируемых напряжений [19].

Образцы помещались в криостат, температура в котором регулировалась изменением потока паров жидкого гелия.

Часто при вычислении оптической плотности $\alpha(\omega) \cdot d$ в сильнопоглощающих материалах пренебрегают отражением [20]. Формула, учитывающая отражение от образца, предложенная в работе [21], обеспечивает лучшую точность. Поэтому впоследствии спектры пропускания и отражения регистрировались поочередно с помощью монохроматора при нормальном падении на образец оптического излучения от полихроматического источника. Типичная оптическая схема экспериментальной установки описана в работе [22], методика измерений - в [18].

Совместная обработка спектров пропускания и отражения становится необходимой в случае возникновения в спектрах модуляции осцилляциями интерференции Фабри-Перо, вызванной отражениями от внутренних гетерограниц многослойных образцов с точно выдержанной толщиной слоев.

Толщина внутреннего слоя $d$ полупроводниковых кристаллов с интерфейсной границей контролируется в процессе роста, что позволяет с высокой точностью определить коэффициент поглощения $\alpha(\omega)$ из измеренной оптической плотности.

Точное определение толщины исследуемого участка открытой пленки образца полупроводникового кристалла, полученного утончением, как правило, приводит к его разрушению и невозможности воспроизведения экспериментального исследования.

\section{3. Температурно-зависимое экситонное поглощение полупроводниковых кристаллов}

На рис. 1 показана температурная эволюция края фундаментального поглощения исследованных полупроводниковых кристаллов InP, GaAs, CdTe.

С увеличением температуры спектральное положение максимума основного экситонного состояния в исследованных полупроводниковых кристаллах смещается в длинноволновую область, следуя за расчетным положением запрещенной зоны $E_{g}(T)$ от температуры в аппроксимации Варшни, что позволяет говорить о сохранении экситонного характера поглощения вплоть до высоких температур.

На примере полупроводникового кристалла GaAs на рис. 2 показаны экспериментальные температурные зависимости интегрального поглощения $K(T)$, максималь-

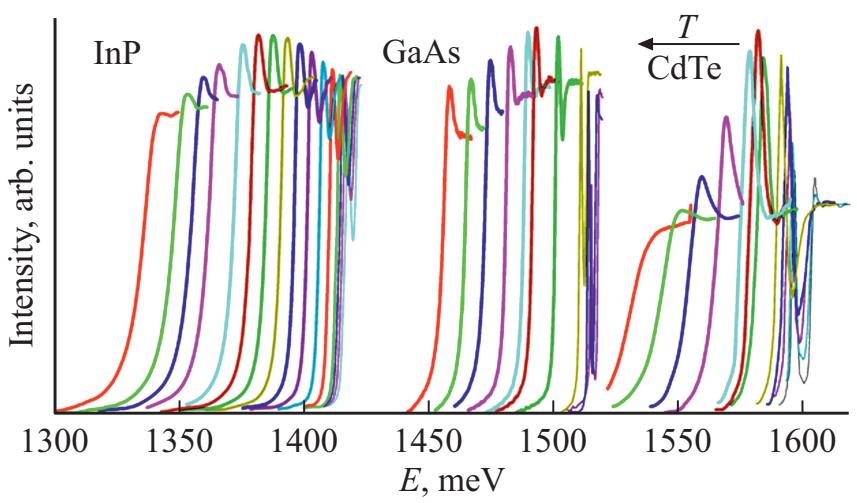

Рис. 1. Температурная эволюция края фундаментального поглощения полупроводниковых кристаллов InP, GaAs, CdTe. Спектры приведены к максимальному значению для каждого полупроводника. 
Экспериментально определенные значения критической температуры, максимального интегрального экситонного поглощения исследованных полупроводниковых кристаллов $\mathrm{GaAs}, \mathrm{InP}, \mathrm{CdTe}, \mathrm{ZnTe}, \mathrm{ZnSe}$ и вычисленные по нему значения продольнопоперечного расщепления и критического параметра затухания экситонных поляритонов в сравнении с подборкой из работ [23,24]

\begin{tabular}{c|c|c|c|c|c|c|c}
\hline \multirow{2}{*}{$\begin{array}{c}\text { Полупроводниковые } \\
\text { кристаллы }\end{array}$} & \multirow{2}{*}{$R_{\mathrm{ex}}$, мэВ } & $T_{c}, \mathrm{~K}$ & $K_{\max }$, эB/см & \multicolumn{2}{|c|}{$\hbar \omega_{L T}$, мэB } & \multicolumn{2}{|c}{$\Gamma_{c}$, мэB } \\
\cline { 3 - 7 } & & эксперимент & эксперимент & эксперимент & {$[23]$} & эксперимент & {$[24]$} \\
\hline $\mathrm{GaAs}$ & 4.1 & 135 & 73 & 0.082 & 0.08 & 0.250 & 0.240 \\
$\mathrm{InP}$ & 5.0 & 200 & 149 & 0.175 & 0.14 & 0.324 \\
$\mathrm{CdTe}$ & 10.7 & 80 & 415 & 0.450 & 0.40 & 0.340 \\
$\mathrm{ZnTe}$ & 12.8 & 110 & 675 & 0.650 & 0.80 & 0.750 \\
$\mathrm{ZnSe}$ & 20.0 & 195 & 1018 & 1.600 & 1.45 & 1.061 & 0.300 \\
& & & &
\end{tabular}

ного коэффициента поглощения $\alpha(T)$ и наблюдаемой ширины линии поглощения $H(T)$ основного экситонного состояния.

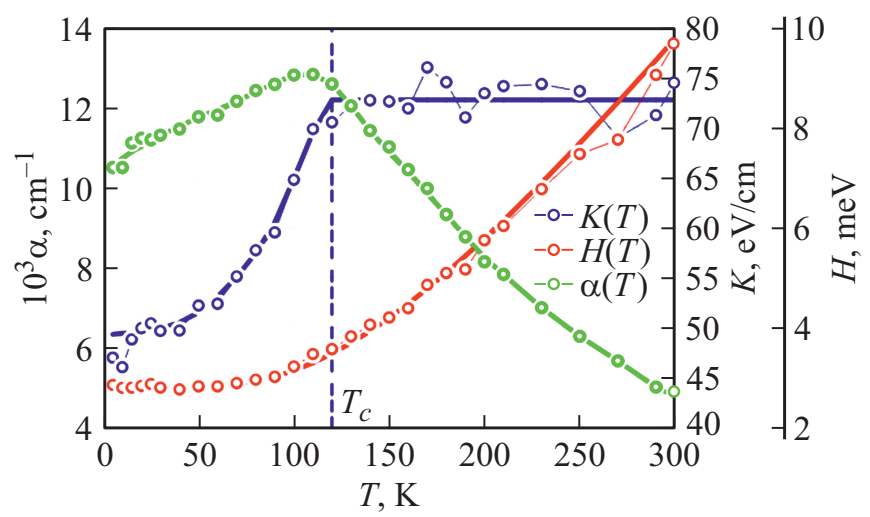

Рис. 2. Экспериментальные температурные зависимости интегрального поглощения $K(T)$, максимального коэффициента поглощения $\alpha(T)$ и наблюдаемой ширины линии поглощения $H(T)$ основного экситонного состояния образца полупроводникового кристалла $\mathrm{GaAs}$ со свободной границей.

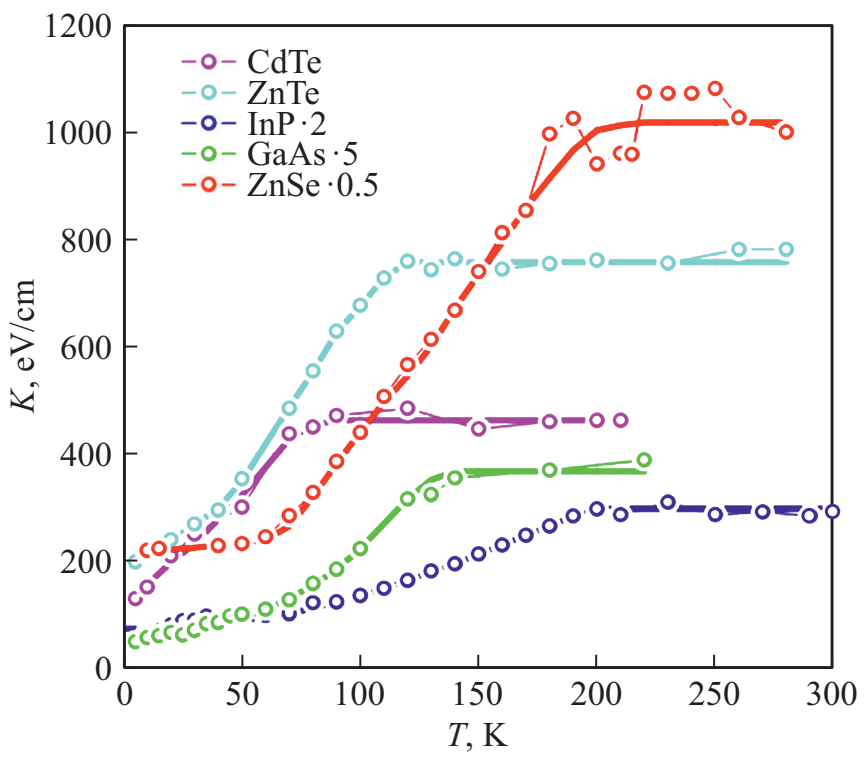

Рис. 3. Экспериментальные температурные зависимости интегрального экситонного поглощения исследованных полупроводниковых кристаллов CdTe, ZnTe, InP, GaAs, ZnSe.
На рис. 3 объединены экспериментальные температурные зависимости интегрального экситонного поглощения $K(T)$ полупроводниковых кристаллов $\mathrm{GaAs}, \mathrm{CdTe}$, $\mathrm{ZnTe}, \mathrm{ZnSe}, \mathrm{InP}$.

Во всех исследованных полупроводниковых кристаллах с увеличением температуры интегральное поглощение $K(T)$ растет до критической температуры $T_{c}$, после которой становится постоянным, принимая свое максимальное значение $K\left(T>T_{c}\right)=K_{\max }$. Значение максимального коэффициента поглощения $\alpha(T)$ также первоначально растет с увеличением температуры, а затем убывает так, чтобы при увеличении наблюдаемой ширины линии $H(T)$ поддерживалось постоянство интегрального поглощения.

Экспериментально определенное максимальное значение интегрального поглощения $K_{\max }$ позволяет вычислить [1] значение энергии продольно-поперечного расщепления $\hbar \omega_{L T}$,

$$
\hbar \omega_{L T}=K_{\max } \frac{c \hbar}{\pi \sqrt{\varepsilon_{b}} \cdot \hbar \omega_{0}},
$$

и значение критического параметра диссипативного затухания,

$$
\Gamma_{c}=2 \hbar \omega_{0} \sqrt{2 \varepsilon_{b} \frac{\hbar \omega_{L T}}{M c^{2}}},
$$

где $\hbar \omega_{0}-$ энергия экситонного резонанса, $\varepsilon_{b}-$ фоновая диэлектрическая проницаемость, $c-$ скорость света, $M$ - трансляционная масса экситона.

Максимальные значения интегрального экситонного поглощения исследованных полупроводниковых кристаллов и определенные по ним параметры экситонных поляритонов приведены в таблице.

\section{4. Заключение}

Проведенные экспериментальные исследования температурно-зависимого интегрального поглощения полупроводниковых кристаллов демонстрируют качественное и количественное соответствие экспериментальных данных с расчетами анализа [1] и свидетельствуют об экситон-поляритонном характере светопереноса в области края фундаментального поглощения. Критерием существования экситонных поляритонов является наличие 
участка монотонного роста интегрального поглощения в области низких температур, соответствующих значениям параметра затухания ниже критического.

Экспериментально определенные значения продольнопоперечного расщепления и критического параметра затухания имеют высокую точность, так как их расчет производится по постоянному значению интегрального поглощения $K\left(T>T_{c}\right)=K_{\max }$ при температурах выше критических $T>T_{c}$.

Для экспериментально исследованных образцов определены критические температуры экситон-поляритонного светопереноса, которые могут быть использованы при создании экситон-поляритонных приборов.

Методом интегрального поглощения диссипативное затухание экситонных поляритонов также изучалось при воздействии факторов магнитного и электрического полей, концентрации введенной или остаточной примеси, толщины образца [25], при этом температурно-зависимое интегральное поглощение можно рассматривать как один из центральных и наиболее общих случаев.

\section{Конфликт интересов}

Авторы заявляют об отсутствии конфликта интересов.

\section{Список литературы}

[1] Н.Н. Ахмедиев. ЖЭТФ, 79, 1534 (1980).

[2] С.И. Пекар. ЖЭТФ, 33, 1022 (1957).

[3] J.J. Hopfield, D.G. Tomas. Phys. Rev., 132 (2), 563 (1963).

[4] А.С. Давыдов. Теория твердого тела (М., Наука, 1976).

[5] J. Voigt. Phys. Status Solidi B, 64 (2), 549 (1974).

[6] Н.Н. Ахмедиев, Г.П. Голубев, В.С. Днепровский, Е.А. Жуков. ФТТ, 25 (7), 2225 (1983).

[7] V.A. Kosobukin, R.P. Seisyan, S.A. Vaganov. Semicond. Sci. Techn., 8, 1235 (1993).

[8] С.А. Ваганов, Р.П. Сейсян. Письма ЖТФ, 38 (19), 9 (2012).

[9] G.N. Aliev, O.S. Coschug-Toates, V.A. Kosobukin, R.P. Seisyan, S.A. Vaganov. Proc. SPIE, 1985, 794 (1993).

[10] R.M. Datsiev, V.A. Kosobukin, N.V. Luk'yanova, R.P. Seisyan, M.R. Vladimirova. Proc. 3rd Int. Symp. on Excitonic Processes in Condensed Matter (EXCON'98), 98-25, 228 (1998).

[11] R. Passler, E. Griebl, H. Riepl, G. Lautner, S. Bauer, H. Preis, W. Gebhardt, B. Buda, D.J. As, D. Schikora, K. Lischka, K. Papagelis, S. Ves. J. App. Phys., 86 (8), 4403 (1999).

[12] M.A. Osman, A.A. Othman. J. Phys. B: Condens. Matter, 239 (3), 231 (1997).

[13] A.J. Fischer, W. Shan, J.J. Song, Y.C. Chang, R. Horning, B. Goldenberg. Appl. Phys. Lett., 71, 1981 (1997).

[14] I.S. Gorban', A.P. Krokhmal', Z.Z. Yanchuk. Phys. Solid State, 42 (9), 1625 (2000).

[15] V.M. Kramar, N.K. Kramar, B.M. Nitsovich. Proc. SPIE (6th Intern. Conf. on Correlation Optics), 5477, 242 (2004).

[16] С.А. Ваганов, Р.П. Сейсян. Письма ЖТФ, 38 (3), 39 (2012).

[17] R.P. Seisyan, V.A. Kosobukin, S.A. Vaganov, M.A. Markosov, T.S. Shamirzaev, K.S. Zhuravlev, A.K. Bakarov, A.I. Toropov. Phys. Status Solidi C, 2 (2), 900 (2005).

[18] С.А. Ваганов, Р.П. Сейсян. ФТП, 45 (1), 104 (2011).
[19] Р.П. Сейсян. Спектроскопия диамагнитных экситонов (М., Наука, 1984)

[20] A.R. Goi, A. Cantarero, K. Syassen, M. Cardona. Phys. Rev. B, 41 (14), 10111 (1990).

[21] D.S. Gerber, G.N. Maracas. IEEE J. Quant. Electron., 29 (10), 2589 (1993).

[22] С.А. Марков, Р.П. Сейсян, В.А. Кособукин. ФТП, 38 (2), 230 (2004).

[23] C. Weisbuch, R.G. Ulbrich. Light Scattering in Solids III: Recent Results, ed. by M. Cardona, G. Güntherodt (BerlinHeidelberg, Springer, 1982) p. 207.

[24] S. Rudin, T.L. Reinecke, B. Segall. Phys. Rev. B, 42 (17), 11218 (1990).

[25] Р.П. Сейсян. ФТП, 58 (5), 833 (2016).

Редактор А.Н. Смирнов

\section{Temperature-dependent integrated absorption of excitonic polaritons in bulk semiconductors}

\author{
R.P. Seisyan, S.A. Vaganov \\ loffe Institute, \\ 194021 St. Petersburg, Russia
}

\begin{abstract}
The given paper highlights the temperature factor in the experimental study of untegral absorption regarded as a response function of excitonic polaritons and the method of experimental confirmation and investigation of the excitonpolaritonic nature of the light transfer near the fundamental absorption edge. The results of experimental studies of the temperature-dependent integrated excitonic absorption of bulk semiconductors are examined and summarized with estimated physical properties of materials (CdTe, GaAs, InP, ZnSe, ZnTe) investigated by the authors.
\end{abstract}

